\title{
REGULARITY OF THE MULTI-CONFIGURATION TIME-DEPENDENT HARTREE APPROXIMATION IN QUANTUM MOLECULAR DYNAMICS*
}

\author{
Othmar KOCH ${ }^{1}$ AND Christian Lubich ${ }^{1}$
}

\begin{abstract}
We discuss the multi-configuration time-dependent Hartree (MCTDH) method for the approximation of the time-dependent Schrödinger equation in quantum molecular dynamics. This method approximates the high-dimensional nuclear wave function by a linear combination of products of functions depending only on a single degree of freedom. The equations of motion, obtained via the Dirac-Frenkel time-dependent variational principle, consist of a coupled system of low-dimensional nonlinear partial differential equations and ordinary differential equations. We show that, with a smooth and bounded potential, the MCTDH equations are well-posed and retain high-order Sobolev regularity globally in time, that is, as long as the density matrices appearing in the method formulation remain invertible. In particular, the solutions are regular enough to ensure local quasi-optimality of the approximation and to admit an efficient numerical treatment.
\end{abstract}

Mathematics Subject Classification. 35F25, 58J90, 81V55.

Received: July 1, 2005.

\section{INTRODUCTION}

This paper deals with an approach to the approximate solution of the time-dependent Schrödinger equation

$$
\mathrm{i} \frac{\partial \psi}{\partial t}=H \psi
$$

where the wave function $\psi=\psi\left(x^{(1)}, \ldots, x^{(d)}, t\right)$ depends on the spatial coordinates $x^{(k)} \in \mathbb{R}^{3}$ of $d$ particles, and on time $t$. In atomic units, the Hamiltonian is given by

$$
H=T+V=-\frac{1}{2 m_{1}} \Delta^{(1)}-\cdots-\frac{1}{2 m_{d}} \Delta^{(d)}+V\left(x^{(1)}, \ldots, x^{(d)}\right),
$$

where in the kinetic energy operator $T$, the Laplacian $\Delta^{(k)}$ is with respect to the spatial coordinates of the $k$ th particle of mass $m_{k}$. The real potential $V$ acts as a multiplication operator. We will assume that $V$ is smooth and bounded.

Keywords and phrases. MCTDH method, wavepacket propagation, nonlinear evolution equation, well-posedness, regularity.

* Supported by DFG, Special Research Program SFB 382.

${ }^{1}$ Universität Tübingen, Mathematisches Institut, Auf der Morgenstelle 10, 72076 Tübingen, Germany. othmar@othmar-koch. org; lubich@na.uni-tuebingen.de 
The applications we have in mind are models from quantum molecular dynamics, where the particles are distinguishable nuclei moving in a potential given by an electronic energy surface, according to the BornOppenheimer approximation to the full molecular Schrödinger equation. The extension to non-adiabatic systems, in the presence of conical intersections of potential energy surfaces, is accommodated by considering vector-valued wave functions with components corresponding to different electronic states, and matrix-valued potentials coupling the electronic states; see $[2,11]$. The assumption of a smooth and bounded potential is usually a reasonable modelling assumption in these applications.

For such problems of molecular dynamics, the multi-configuration time-dependent Hartree (MCTDH) method has been put forward by H.-D. Meyer and coauthors in $[3,4,20,21]$ and further references therein. It has been used with great success for computational wavepacket propagation in a variety of chemical situations such as photodissociation and reactive scattering, for problems involving 6 to 24 nuclear degrees of freedom and one or several electronic states; see, e.g., [23]. In the MCTDH approach, the wave function is approximated by a linear combination of Hartree products, that is, of products of functions each depending on the coordinates of only a single particle, or of a single degree of freedom. The Dirac-Frenkel time-dependent variational principle yields equations of motion for the single-particle functions and the coefficients in the linear combination of the Hartree products. The MCTDH method thus replaces the high-dimensional linear Schrödinger equation by a system of low-dimensional nonlinear partial differential equations and ordinary differential equations and in this way makes the problem computationally tractable.

In this paper we study the nonlinear equations of motion of the MCTDH approach. For initial data in the Sobolev space $H^{2}$ of functions that are square integrable together with all their first and second-order partial derivatives, we show that a unique solution to the MCTDH equations exists in $H^{2}$ as long as the core tensor of the method is of full rank, or equivalently, as long as the density matrices of the method remain nonsingular. Our arguments also yield $H^{2}$ Lipschitz continuous dependence of the solution on the initial data. The global regularity and well-posedness result extends to $H^{m}$ for arbitrary $m \geq 2$ and to higher temporal regularity if the initial data is in $H^{m}$ and the potential is sufficiently smooth. The modified MCTDH equations with regularised density matrices have solutions in $H^{m}$ for all times. The regularity has important consequences for the approximation properties of the approach:

- In [18], the $H^{2}$ regularity is essential for showing quasi-optimality in the sense that the error of the variational approximation is bounded by a (time-dependent) multiple of the distance of the exact wave function to the approximation manifold.

- The second-order convergence of the variational splitting numerical time integration scheme of [17] depends on $H^{2}$ spatial regularity of the solution. Second and higher order space discretisations require regularity in $H^{m}$ with $m \geq 2$.

In Section 1, we give a mathematical description of the MCTDH method, and in Section 2 we state our results concerning well-posedness and regularity of the MCTDH equations, which are proven in Section 3. Finally, Section 4 concludes the paper with further remarks.

Let us mention some mathematical works treating related models and problems: Bove et al. $[5,6]$ and Chadam and Glassey [9] study the global existence of solutions to the (single-configuration) time-dependent HartreeFock equations under varying assumptions on the potential. Friesecke [14] proves the existence of ground state solutions for the (stationary) multiconfiguration self-consistent field equations with Coulomb potential, and Lewin [16] studies excited states for these equations.

\section{THE MCTDH METHOD}

In the MCTDH method [4,21], the multi-particle wave function $\psi$ is approximated by a linear combination of Hartree products, that is, for $x=\left(x^{(1)}, \ldots, x^{(d)}\right)$,

$$
\psi(x, t) \approx u(x, t)=\sum_{J} a_{J}(t) \Phi_{J}(x, t)=\sum_{\left(j_{1}, \ldots, j_{d}\right)} a_{j_{1}, \ldots, j_{d}}(t) \phi_{j_{1}}^{(1)}\left(x^{(1)}, t\right) \cdots \phi_{j_{d}}^{(d)}\left(x^{(d)}, t\right) .
$$


Here, the multi-indices $J=\left(j_{1}, \ldots, j_{d}\right)$ vary for $j_{k}=1, \ldots, n_{k}, k=1, \ldots, d$, the $a_{J}(t)$ are complex coefficients depending only on $t$, and the single-particle functions $\phi_{j_{k}}^{(k)}\left(x^{(k)}, t\right)$ depend on the coordinates $x^{(k)}$ of a single particle and on time $t$. This is a model reduction analogous to low-rank approximation of matrices, where a large system matrix is replaced by a linear combination of rank-1 matrices $v \otimes w$.

The Dirac-Frenkel time-dependent variational principle $[10,13]$ is used to derive differential equations for the coefficients $a_{J}$ and the single-particle functions $\phi_{j_{k}}^{(k)}$. We first recall this variational approximation procedure in its abstract form and then turn to the MCTDH approximation manifold and the MCTDH equations of motion.

\subsection{The Dirac-Frenkel variational approximation principle}

The abstract setting is that of the time-dependent Schrödinger equation

$$
\mathrm{i} \frac{\mathrm{d} \psi}{\mathrm{d} t}=H \psi, \quad \psi(0)=\psi_{0}
$$

where the Hamiltonian $H$ is a self-adjoint linear operator on a complex Hilbert space $\mathcal{H}$ with inner product $\langle\cdot \mid \cdot\rangle$ and norm $\|\cdot\|$. Let $\mathcal{M} \subset \mathcal{H}$ be a manifold on which an approximation to the wave function $\psi(t)$ should lie, and let $\mathcal{T}_{u} \mathcal{M}$ denote the tangent space at $u \in \mathcal{M}$ (i.e., the closed real-linear subspace of $\mathcal{H}$ formed of the derivatives of all paths on $\mathcal{M}$ passing through $u$, or in physical terminology, the space of admissible variations). We assume that $\mathcal{T}_{u} \mathcal{M}$ is in fact complex linear, i.e., with $\delta u \in \mathcal{T}_{u} \mathcal{M}$ also i $\delta u \in \mathcal{T}_{u} \mathcal{M}$. The Dirac-Frenkel principle determines the approximate wave function $t \mapsto u(t) \in \mathcal{M}$ from the condition [10] that the time derivative should satisfy, at every time $t$,

$$
\left\langle\delta u \mid \frac{\mathrm{d} u}{\mathrm{~d} t}-\frac{1}{\mathrm{i}} H u\right\rangle=0 \quad \text { for all } \quad \delta u \in \mathcal{T}_{u} \mathcal{M} .
$$

Since we assume $\mathcal{T}_{u} \mathcal{M}$ to be complex linear, this condition remains unchanged if only the real part or only the imaginary part is taken. This leads to two different interpretations:

1) Taking the real part yields the interpretation as an orthogonal projection: with the orthogonal projection $P(u): \mathcal{H} \rightarrow \mathcal{T}_{u} \mathcal{M}$ given by $\operatorname{Re}\langle\delta u \mid P(u) \varphi\rangle=\operatorname{Re}\langle\delta u \mid \varphi\rangle$ for all $\delta u \in \mathcal{T}_{u} \mathcal{M}$ and $\varphi \in \mathcal{H}$, condition (5) amounts to projecting the vector field at $u$ to the tangent space at $u$,

$$
\frac{\mathrm{d} u}{\mathrm{~d} t}=P(u) \frac{1}{\mathrm{i}} H u .
$$

It follows (see [13], p. 253, and [19]) that $\mathrm{d} u / \mathrm{d} t$ is the solution of the minimisation problem to choose

$$
\frac{\mathrm{d} u}{\mathrm{~d} t}=\arg \min _{\vartheta \in \mathcal{T}_{u} \mathcal{M}}\left\|\vartheta-\frac{1}{\mathrm{i}} H u\right\| .
$$

The interpretation as an orthogonal projection is essential for showing quasi-optimality of the variational approximation [18]. Taking the real part in (5) also yields that conserved quantities of the Schrödinger equation are preserved if they map into the tangent space: if a self-adjoint operator $A$ commutes with the Hamiltonian $H$ and if $A u \in \mathcal{T}_{u} \mathcal{M}$ for all $u \in \mathcal{M}$, then $\langle u(t)|A| u(t)\rangle=$ Const.; see [12]. In particular, taking $A$ as the identity operator shows that for manifolds with $u \in \mathcal{T}_{u} \mathcal{M}$ (which is the case if, with $u \in \mathcal{M}$, also scalar multiples of $u$ are in $\mathcal{M})$, there is conservation of norm, $\|u(t)\|=$ Const.; see also [4].

2) Taking the imaginary part yields the interpretation as a symplectic projection: consider the symplectic two-form on $\mathcal{H}$ given by $\omega(\xi, \eta)=\operatorname{Im}\langle\xi \mid \eta\rangle$. The complex linearity of $\mathcal{T}_{u} \mathcal{M}$ ensures that $\mathcal{M}$ is a symplectic submanifold of $\mathcal{H}$, that is, the symplectic two-form $\omega$ is non-degenerate. With the symplectic projection $\Pi(u): \mathcal{H} \rightarrow \mathcal{T}_{u} \mathcal{M}$ given by $\omega(\delta u, \Pi(u) \varphi)=\omega(\delta u, \varphi)$ for all $\delta u \in \mathcal{T}_{u} \mathcal{M}$ and $\varphi \in \mathcal{H}$, taking the imaginary part in condition (5) amounts to

$$
\mathrm{i} \frac{\mathrm{d} u}{\mathrm{~d} t}=\Pi(u) H u
$$


It then follows that the differential equations in coordinates on $\mathcal{M}$ become a non-canonical Hamiltonian system $[12,24]$. As a consequence, the symplectic two-form $\omega$ restricted to the tangent space is conserved along the flow, and the total energy $\langle u(t)|H| u(t)\rangle$ is conserved. (The latter is also seen directly by choosing $\delta u=\mathrm{d} u / \mathrm{d} t$ in (5) and taking the imaginary part.)

We finally note that, from a numerical analysis viewpoint, condition (5) can be seen as a Galerkin condition on the solution-dependent tangent space $\mathcal{T}_{u} \mathcal{M}$.

\subsection{The MCTDH approximation manifold}

The MCTDH method determines approximations to the wave function that, for every time $t$, lie in the set

$$
\overline{\mathcal{M}}=\left\{u \in L^{2}\left(\mathbb{R}^{D}\right): u=\sum_{J} a_{J} \phi_{j_{1}}^{(1)} \otimes \cdots \otimes \phi_{j_{d}}^{(d)} \quad \text { with } a_{J} \in \mathbb{C}, \phi_{j_{k}}^{(k)} \in L^{2}\left(\mathbb{R}^{D_{k}}\right)\right\}
$$

with multi-indices $J=\left(j_{1}, \ldots, j_{d}\right)$ ranging over $j_{k}=1, \ldots, n_{k}$, and the total dimension $D=D_{1}+\cdots+D_{d}$. This set $\overline{\mathcal{M}}$ is not a manifold, but as we now explain, $\overline{\mathcal{M}}$ contains a dense subset $\mathcal{M}$ that is a manifold and is characterised by a full-rank condition.

A difficulty lies in the fact that the representation of $u \in \overline{\mathcal{M}}$ by coefficients $a=\left(a_{J}\right)_{J}$ and single-particle functions $\phi=\left(\phi_{j_{k}}^{(k)}\right)_{j_{k}, k}$ is not unique. In fact, the change of variables

$$
\begin{aligned}
& \phi_{j_{k}}^{(k)} \rightarrow \widehat{\phi}_{j_{k}}^{(k)}=\sum_{l_{k}=1}^{n_{k}} S_{j_{k}, l_{k}}^{(k)} \phi_{l_{k}}^{(k)}, \\
& a_{J} \rightarrow \widehat{a}_{J}=\sum_{i_{1}=1}^{n_{1}} \cdots \sum_{i_{d}=1}^{n_{d}}\left(S^{(1)}\right)_{i_{1}, j_{1}}^{-1} \cdots\left(S^{(d)}\right)_{i_{d}, j_{d}}^{-1} a_{I}
\end{aligned}
$$

defines the same function $u$ for any choice of nonsingular matrices $S^{(1)}, \ldots, S^{(d)}$. We may, however, assume that the $\phi_{j_{k}}^{(k)}$ corresponding to the same particle $k$ are orthonormal:

$$
\left\langle\phi_{j_{k}}^{(k)} \mid \phi_{l_{k}}^{(k)}\right\rangle=\delta_{j_{k}, l_{k}}, \quad j_{k}, l_{k}=1, \ldots, n_{k}, k=1, \ldots, d
$$

(where $\delta_{j_{k}, l_{k}}$ is Kronecker's delta). This still does not make the representation unique, but by associating a particular representation $(a, \phi)$ to a selected point $u \in \overline{\mathcal{M}}$, we now aim at finding a uniquely determined nearby representation for nearby points on $\overline{\mathcal{M}}$. Consider a differentiable path $t \mapsto(a(t), \phi(t))$ representing a path $u(t)$ on $\overline{\mathcal{M}}$. Then, the derivative $\delta u=\dot{u}(0)$ is of the form

$$
\delta u=\sum_{J} \delta a_{J} \Phi_{J}+\sum_{k=1}^{d} \sum_{j_{k}=1}^{n_{k}} \delta \phi_{j_{k}}^{(k)} \psi_{j_{k}}^{(k)}
$$

with the Hartree products $\Phi_{J}=\bigotimes_{k=1}^{d} \phi_{j_{k}}^{(k)}$ and with the single-hole functions

$$
\psi_{j_{k}}^{(k)}:=\left\langle\phi_{j_{k}}^{(k)} \mid u\right\rangle^{(k)}=\sum_{j_{1}=1}^{n_{1}} \cdots \sum_{j_{k-1}=1}^{n_{k-1}} \sum_{j_{k+1}=1}^{n_{k+1}} \cdots \sum_{j_{d}=1}^{n_{d}} a_{j_{1}, \ldots, j_{d}} \bigotimes_{\ell \neq k} \phi_{j_{\ell}}^{(\ell)}
$$

where the superscript $(k)$ on the inner product indicates that the $L^{2}$ inner product is taken only with respect to the variable $x^{(k)}$, leaving a function depending on all the other variables $x^{(\ell)}$ with $\ell \neq k$. Conversely, the $\delta a_{J}$ are uniquely determined by $\delta u$ and $(a, \phi)$ if we impose the orthogonality constraint

$$
\left\langle\phi_{j_{k}}^{(k)} \mid \delta \phi_{l_{k}}^{(k)}\right\rangle=0, \quad j_{k}, l_{k}=1, \ldots, n_{k}, k=1, \ldots, d
$$


which together with (12) implies

$$
\delta a_{J}=\left\langle\Phi_{J} \mid \delta u\right\rangle .
$$

Taking the inner product of (13) with $\psi_{i_{k}}^{(k)}$ then gives

$$
\sum_{j_{k}=1}^{n_{k}} \rho_{i_{k}, j_{k}}^{(k)} \delta \phi_{j_{k}}^{(k)}=\left\langle\psi_{i_{k}}^{(k)} \mid \delta u-\sum_{J} \delta a_{J} \Phi_{J}\right\rangle^{(\neg k)}
$$

with the hermitian, positive semi-definite density matrices

$$
\rho^{(k)}=\left(\rho_{i_{k}, j_{k}}^{(k)}\right)_{i_{k}, j_{k}=1}^{n_{k}} \quad \text { given by } \quad \rho_{i_{k}, j_{k}}^{(k)}:=\left\langle\psi_{i_{k}}^{(k)} \mid \psi_{j_{k}}^{(k)}\right\rangle .
$$

The superscript $(\neg k)$ indicates that the $L^{2}$ inner product is taken over all variables except $x^{(k)}$, leaving a function depending on $x^{(k)}$. The orthonormality relations (12) allow us to express the entries of the density matrices in terms of the coefficients $a_{J}$ :

$$
\rho_{i_{k}, j_{k}}^{(k)}=\sum_{j_{1}=1}^{n_{1}} \ldots \sum_{j_{k-1}=1}^{n_{k-1}} \sum_{j_{k+1}=1}^{n_{k+1}} \cdots \sum_{j_{d}=1}^{n_{d}} \bar{a}_{j_{1}, \ldots, j_{k-1}, i_{k}, j_{k+1}, \ldots, j_{d}} a_{j_{1}, \ldots, j_{d}} .
$$

The $\delta \phi_{j_{k}}^{(k)}$ are thus uniquely determined from (16), and depend $L^{2}$-continuously on $\delta u$, under the full-rank condition that

$$
\rho^{(k)} \text { is an invertible matrix for each } k=1, \ldots, d \text {. }
$$

(In view of (18), a necessary condition for this property is $n_{k} \leq \prod_{\ell \neq k} n_{\ell}$.)

With the above construction of the $\delta a_{J}$ and $\delta \phi_{j_{k}}^{(k)}$, we obtain local charts on

$$
\begin{aligned}
\mathcal{M}= & \left\{u \in L^{2}\left(\mathbb{R}^{D}\right): u=\sum_{J} a_{J} \phi_{j_{1}}^{(1)} \otimes \cdots \otimes \phi_{j_{d}}^{(d)} \text { with } a_{J} \in \mathbb{C}, \phi_{j_{k}}^{(k)} \in L^{2}\left(\mathbb{R}^{D_{k}}\right)\right. \\
& \text { satisfying the orthonormality constraints (12) and the full-rank condition (19) }\},
\end{aligned}
$$

making this set an infinite-dimensional manifold, for which the tangent space at $u \in \mathcal{M}$ consists of the elements $\delta u$ of the form (13). The MCTDH method is obtained by using the Dirac-Frenkel principle on this approximation manifold $\mathcal{M}$.

We remark that in differential-geometric terminology, the mapping $\pi:(a, \phi) \mapsto u=\sum a_{J} \Phi_{J}$ is a principal bundle with fiber given by the group $G L\left(n_{1}\right) \times \cdots \times G L\left(n_{d}\right)$. The above construction yields a local section $\sigma: u \mapsto(a, \phi)$.

\subsection{The MCTDH equations of motion}

Using the Dirac-Frenkel principle on the approximation manifold $\mathcal{M}$ of (20) and imposing, in view of (15), additional orthogonality constraints on the time derivatives of the single-particle functions $\phi_{j_{k}}^{(k)}\left(x^{(k)}, t\right)$,

$$
\left\langle\phi_{j_{k}}^{(k)} \mid \frac{\partial \phi_{l_{k}}^{(k)}}{\partial t}\right\rangle=0, \quad t \geq 0, \quad j_{k}, l_{k}=1, \ldots, n_{k}, k=1, \ldots, d
$$


yields a system of coupled ordinary and partial differential equations for the coefficients and single-particle functions, rigorously derived in $[4,21]$ under the implicit assumption that a sufficiently regular solution exists:

$$
\begin{aligned}
\mathrm{i} \frac{\mathrm{d} a_{J}}{\mathrm{~d} t} & =\sum_{K}\left\langle\Phi_{J}|H| \Phi_{K}\right\rangle a_{K}, \quad \forall J \\
\mathrm{i} \frac{\partial \phi_{j_{k}}^{(k)}}{\partial t} & =\left(1-P^{(k)}\right) \sum_{m_{k}=1}^{n_{k}} \sum_{l_{k}=1}^{n_{k}}\left(\rho^{(k)}\right)_{j_{k}, m_{k}}^{-1}\left\langle\psi_{m_{k}}^{(k)}|H| \psi_{l_{k}}^{(k)}\right\rangle^{(\neg k)} \phi_{l_{k}}^{(k)}, \quad j_{k}=1, \ldots, n_{k}, k=1, \ldots, d,
\end{aligned}
$$

where the Hartree products $\Phi_{J}$, the single-hole functions $\psi_{l_{k}}^{(k)}$, and the density matrices $\rho^{(k)}$ are defined as in Section 1.2, where the superscript $(\neg k)$ on the inner product indicates that the inner product is over all variables except $x^{(k)}$, and where $P^{(k)}$ is the orthogonal projector onto the space spanned by $\phi_{1}^{(k)}, \ldots, \phi_{n_{k}}^{(k)}$,

$$
P^{(k)} \varphi=\sum_{j_{k}=1}^{n_{k}} \phi_{j_{k}}^{(k)}\left\langle\phi_{j_{k}}^{(k)} \mid \varphi\right\rangle^{(k)}
$$

The choice of the constraint (21) is not mandatory. The orthonormality constraints (12) imply only the weaker condition

$$
\frac{\mathrm{d}}{\mathrm{d} t}\left\langle\phi_{j_{k}}^{(k)} \mid \phi_{l_{k}}^{(k)}\right\rangle=\left\langle\frac{\partial \phi_{j_{k}}^{(k)}}{\partial t} \mid \phi_{l_{k}}^{(k)}\right\rangle+\left\langle\phi_{j_{k}}^{(k)} \mid \frac{\partial \phi_{l_{k}}^{(k)}}{\partial t}\right\rangle=0
$$

In fact, any constraint of the form

$$
\left\langle\phi_{j_{k}}^{(k)} \mid \frac{\partial \phi_{l_{k}}^{(k)}}{\partial t}\right\rangle=-\mathrm{i}\left\langle\phi_{j_{k}}^{(k)}\left|G^{(k)}\right| \phi_{l_{k}}^{(k)}\right\rangle, \quad t \geq 0, \quad j_{k}, l_{k}=1, \ldots, n_{k}, k=1, \ldots, d,
$$

with self-adjoint operators $G^{(k)}$ serves to define a unique solution satisfying the orthonormality constraints (12). The formulation of the differential equations in case of general self-adjoint operators $G^{(k)}$ is given in [4]. Changing the constraint operators $G^{(k)} \rightarrow \widetilde{G}^{(k)}$ in (25) generates a transformation (10), (11) where the time-dependent matrices $S^{(k)}(t)$ satisfy (see [8])

$$
\mathrm{i} \frac{\mathrm{d}}{\mathrm{d} t} S^{(k)}=F^{(k)} S^{(k)}, \quad \text { where } \quad F^{(k)}=\left(F_{j_{k}, l_{k}}^{(k)}\right) \quad \text { with } \quad F_{j_{k}, l_{k}}^{(k)}=\left\langle\phi_{j_{k}}^{(k)}\left|\widetilde{G}^{(k)}-G^{(k)}\right| \phi_{l_{k}}^{(k)}\right\rangle .
$$

For given $\phi_{j_{k}}^{(k)}$, this system of linear ordinary differential equations together with the initial conditions $S^{(k)}(0)=I$ has a unique solution. Since the matrices $F^{(k)}(t)$ are hermitian, the matrices $S^{(k)}(t)$ remain unitary for all times. It follows that it is sufficient to discuss the solution of the MCTDH equations for a particular choice of the constraints (25), the questions of solvability and regularity of the solution of the differential equations resulting from (25) for any choice of self-adjoint operators $G^{(k)}$ being equivalent.

For our analysis it turns out that the choice $G^{(k)}=T^{(k)}=-\frac{1}{2 m_{k}} \Delta^{(k)}$ is most favourable. In this case the MCTDH equations of motion become

$$
\begin{aligned}
\mathrm{i} \frac{\mathrm{d} a_{J}}{\mathrm{~d} t} & =\sum_{K}\left\langle\Phi_{J}|V| \Phi_{K}\right\rangle a_{K}, \quad \forall J \\
\mathrm{i} \frac{\partial \phi_{j_{k}}^{(k)}}{\partial t} & =T^{(k)} \phi_{j_{k}}^{(k)}+\left(1-P^{(k)}\right) \sum_{l_{k}=1}^{n_{k}} \sum_{m_{k}=1}^{n_{k}}\left(\rho^{(k)}\right)_{j_{k}, m_{k}}^{-1}\left\langle\psi_{m_{k}}^{(k)}|V| \psi_{l_{k}}^{(k)}\right\rangle^{(\neg k)} \phi_{l_{k}}^{(k)}, \quad j_{k}=1, \ldots, n_{k}, k=1, \ldots, d .
\end{aligned}
$$

Compared with (22)-(23), they offer the advantage that the first equation contains only the bounded potential $V$, and in the second equation the kinetic energy operators $T^{(k)}$ appear outside the projection. It is equations (27)(28) that will be analysed in the sequel. 


\section{Statement of Results}

In this section we state the regularity results, which are developed in detail in Section 3.

Theorem 2.1. Consider the system (27)-(28) together with initial conditions chosen such that the orthonormality constraints (12) are satisfied and the density matrices $\rho^{(k)}$ defined in (17) are nonsingular. Assume that the potential $V$ in (2) is bounded and twice continuously differentiable, with bounded first and second derivatives. If the initial data for $\phi_{j_{k}}^{(k)}$ is in the Sobolev space $H^{2}$, then there is a $t^{*}>0$ such that for $t \in\left[0, t^{*}\right)$ there exists a unique classical solution of the MCTDH equations (27)-(28) satisfying

$$
a_{J} \in C^{2}\left(\left[0, t^{*}\right), \mathbb{C}\right), \quad \phi_{j_{k}}^{(k)} \in C^{1}\left(\left[0, t^{*}\right), L^{2}\right) \cap C\left(\left[0, t^{*}\right), H^{2}\right)
$$

The solution can be continued in time until some density matrix $\rho^{(k)}$ becomes singular:

$$
t^{*}=\infty \text { or } \lim _{t \uparrow t^{*}}\left\|\left(\rho^{(k)}(t)\right)^{-1}\right\|=\infty \text { for some } k \text {. }
$$

The solution depends on the initial data Lipschitz continuously with respect to the $H^{2}$ norm on every closed subinterval $[0, \bar{t}]$ with $\bar{t}<t^{*}$. Moreover, for $u$ defined by $a_{J}, \phi_{j_{k}}^{(k)}$ via $(3)$, we have $u(t) \in H^{2}$ for $t \in\left[0, t^{*}\right)$, and $u$ solves the Dirac-Frenkel variational equation (5).

It would be comparatively straightforward to show the stated regularity locally on some interval $\left[0, t_{2}\right)$ by using a fixed-point iteration in $H^{2}$ (or on $\left[0, t_{m}\right)$ in $H^{m}$ for $m \geq 2$ in Cor. 2.2 below). However, we see no way to extend this local result in a direct way to the global-in-time result stated here, and therefore we proceed differently, using commutator identities as our basic tool.

We first show that a unique mild solution of the system (27)-(28) exists. The mild solution is the solution of a system of integral equations derived from the original system of differential equations via the variation of constants formula, see for example [22]. A simple contraction argument shows that a unique solution $a_{J} \in$ $C\left(\left[0, t_{0}\right), \mathbb{C}\right)$ and $\phi_{j_{k}}^{(k)} \in C\left(\left[0, t_{0}\right), L^{2}\right)$ to the integral equation exists for some $t_{0}>0$, which depends on the norms of $\phi_{j_{k}}^{(k)}$ and $\left(\rho^{(k)}\right)^{-1}$. For this argument, we ignore the orthonormality condition (12) for the single particle functions and formally define the density matrices $\rho^{(k)}$ by $(18)$. To show the regularity of this solution, we formally differentiate the integral equations for $\phi_{j_{k}}^{(k)}$. Next it is shown that unique solutions in $L^{2}$ of these formal equations exist on the whole interval $\left[0, t_{0}\right)$ where the mild solution exists. To this end we use bounds for Lie commutators of the vector field defined by the potential $V$ with partial differential operators. These commutators are introduced and calculated in Lemma 3.1 below. The required estimates for the commutators then follow directly from the smoothness of $V$. To proceed, we use regularisations of the integral equations defining the mild solution. These have regular solutions which converge to the mild solution, and moreover their derivatives converge to the solutions of the formal integral equations. Thus, from the closedness of the differential operators we conclude that the formally derived integral equations indeed define the derivatives of the mild solution. Consequently, the mild solution is in fact a classical solution of (27)-(28) which is in $H^{2}$ on $\left[0, t_{0}\right)$. With this assertion we can show that the orthonormality constraint (12) is indeed satisfied for $t \in\left[0, t_{0}\right)$, and the formal definition (18) of the density matrices corresponds with (17). The now established conservation of the $L^{2}$ norms of the $\phi_{j_{k}}^{(k)}$ then implies that the mild solution actually exists on $\left[0, t^{*}\right)$ with $t^{*}$ as stated in the theorem: $t_{0}=t^{*}$. In total, we then have the $H^{2}$ regularity on $\left[0, t^{*}\right)$. Finally, this implies that $u$ defined in (3) satisfies the variational equations (5).

The same arguments can be used to extend the result to higher regularity if the initial data is sufficiently smooth. This is formulated in the following corollary.

Corollary 2.2. Let the assumptions of Theorem 2.1 hold. In addition, suppose that the potential $V$ in (2) is bounded and $m$ times continuously differentiable with bounded derivatives. If the initial data for $\phi_{j_{k}}^{(k)}$ is in the Sobolev space $H^{m}$ with $m>2$, then the solutions of $(27)-(28)$ satisfy $\phi_{j_{k}}^{(k)}(t) \in H^{m}, t \in\left[0, t^{*}\right)$ with the same $t^{*}$ 
as in Theorem 2.1, and depend Lipschitz continuously on the initial data with respect to the $H^{m}$ norm on every closed subinterval of $\left[0, t^{*}\right)$. This is accompanied by higher temporal regularity: for even $m=2 r$, the solution satisfies

$$
a_{J} \in C^{r+1}\left(\left[0, t^{*}\right), \mathbb{C}\right), \quad \phi_{j_{k}}^{(k)} \in C^{r}\left(\left[0, t^{*}\right), L^{2}\right) \cap C\left(\left[0, t^{*}\right), H^{2 r}\right) .
$$

Consequently, the variational approximation satisfies $u(t) \in H^{m}$, and the function $t \mapsto u(t) \in L^{2}$ is $r$ times continuously differentiable.

In Theorem 2.1 and Corollary 2.2, the existence and regularity of a solution of the MCTDH equations is guaranteed for all times $t$ such that the density matrices $\rho^{(k)}$ defined in (17) are nonsingular. In practice, a regularisation $\rho^{(k)}+\varepsilon I$ with a small $\varepsilon>0$ of the density matrices is used to ensure that the MCTDH equations can be solved numerically without providing especially for the case of singular $\rho^{(k)}$, see [4]. It is clear from the arguments in Section 3 that the resulting modified system can be solved for all times $t \in[0, \infty)$, with a solution in $H^{m}$ (of course, the $H^{m}$ norms may then depend on the regularisation parameter $\varepsilon$ ).

\section{Analysis of the MCTDH equations}

\subsection{Notation}

For our analysis, we consider the MCTDH equations in the form (27)-(28). For convenience, we define $N=\prod_{k=1}^{d} n_{k}$ and $n=\sum_{k=1}^{d} n_{k}$, and denote as

$$
a=\left(a_{J}\right)_{J} \in \mathbb{C}^{N} \quad \text { and } \quad \phi=\left(\phi^{(k)}\right)_{k}=\left(\phi_{j_{k}}^{(k)}\right)_{j_{k}, k} \in\left(L^{2}\right)^{n}
$$

(with $\phi^{(k)} \in\left(L^{2}\right)^{n_{k}}$ ) two vectors containing the coefficients and single-particle functions. Then, our problem can be written (the dot symbolizes differentiation with respect to $t$ )

$$
\begin{aligned}
\dot{a} & =A(\phi) a, \\
\dot{\phi} & =\frac{1}{\mathrm{i}} T \phi+B(a, \phi) \phi,
\end{aligned}
$$

with the complex skew-hermitian $N \times N$ matrix $A$ and the $n \times n$ matrix $B$ with operator-valued entries, given by

$$
\begin{aligned}
A_{J, K}(\phi) & =\frac{1}{\mathrm{i}}\left\langle\Phi_{J}|V| \Phi_{K}\right\rangle \\
B(a, \phi) & =\operatorname{blockdiag}\left(B^{(k)}(a, \phi)\right), \quad k=1, \ldots, d, \\
B_{j_{k}, l_{k}}^{(k)}(a, \phi) & =\frac{1}{\mathrm{i}}\left(1-P^{(k)}\right) \sum_{m_{k}=1}^{n_{k}}\left(\rho^{(k)}\right)_{j_{k}, m_{k}}^{-1}\left\langle\psi_{m_{k}}^{(k)}|V| \psi_{l_{k}}^{(k)}\right\rangle^{(\neg k)} .
\end{aligned}
$$

In the above formulas, $\Phi_{J}=\bigotimes_{k=1}^{d} \phi_{j_{k}}^{(k)}$ with $J=\left(j_{1}, \ldots, j_{d}\right)$ is a Hartree product, and $\psi_{j_{k}}^{(k)}$ is the single-hole function defined by (14). The density matrices $\rho^{(k)}=\rho^{(k)}(a)$ are considered as being defined by (18).

For coefficient vectors $a \in \mathbb{C}^{N}$, we denote the Euclidean norm

$$
\|a\|=\left(\sum_{J}\left|a_{J}\right|^{2}\right)^{1 / 2} .
$$

For the single particle functions $\phi \in\left(L^{2}\right)^{n}$ we use

$$
\|\phi\|=\max _{j_{k}, k}\left\|\phi_{j_{k}}^{(k)}\right\|
$$


where $\left\|\phi_{j_{k}}^{(k)}\right\|$ is the usual $L^{2}$ norm. For the pair $(a, \phi)$, we use the norm

$$
\|(a, \phi)\|=\max \{\|a\|,\|\phi\|\} .
$$

We will consider the differential equations (29)-(30) with initial data $\left(a_{0}, \phi_{0}\right)$ of unit norm,

$$
\left\|\left(a_{0}, \phi_{0}\right)\right\|=1 .
$$

We further assume that the components of $\phi_{0}$ satisfy the orthonormality constraints (12), so that the initial wavefunction $u_{0}=\sum_{J} a_{0, J} \Phi_{0, J}$ is of unit $L^{2}$ norm. We will also assume that the components of $\phi_{0}$ are in the Sobolev space $H^{2}$.

\subsection{Bounds and Lipschitz bounds for the system matrices}

The above norms induce matrix norms $\|A\|,\|B\|$. We now collect bounds and Lipschitz bounds of $A(\phi)$ and $B(a, \phi)$, whose straightforward proofs we omit. For bounded $\|(a, \phi)\| \leq r$, we obtain the bound and Lipschitz bound

$$
\|A(\phi)\| \leq \alpha_{0}, \quad\|A(\phi)-A(\widetilde{\phi})\| \leq \alpha_{1}\|\phi-\widetilde{\phi}\|,
$$

where $\alpha_{0}$ and $\alpha_{1}$ are proportional to $\|V\|=\max _{x}|V(x)|$ and depend on $r$ and on the dimension.

Corresponding bounds for $B$ are obtained in regions of $(a, \phi)$ where the density matrices $\rho^{(k)}=\rho^{(k)}(a)$ defined by (18) have bounded inverses:

$$
\left\|\rho^{-1}\right\|=\max _{k}\left\|\left(\rho^{(k)}\right)^{-1}\right\| \leq M .
$$

We then obtain, for $a$ and $\widetilde{a}$ in a ball where (35) holds,

$$
\|B(a, \phi)\| \leq \beta_{0} M, \quad\|B(a, \phi)-B(\widetilde{a}, \widetilde{\phi})\| \leq \beta_{1} M^{2}\|(a, \phi)-(\widetilde{a}, \widetilde{\phi})\|,
$$

where $\beta_{0}$ and $\beta_{1}$ depend again on $\|V\|$ and $r$.

Next, we consider the directional derivative of $B(a, \phi)$ with respect to $\phi$, which we denote by $B^{\prime}(a, \phi)$. Let $\phi(\tau)$ be a differentiable function satisfying $\phi(0)=\phi, \dot{\phi}(0)=\delta \phi$, then the directional derivative in direction $\delta \phi$ is given by

$$
B^{\prime}(a, \phi) \delta \phi=\left.\frac{\mathrm{d}}{\mathrm{d} \tau}\right|_{\tau=0} B(a, \phi(\tau))
$$

On noting that

$$
\left.\frac{\mathrm{d}}{\mathrm{d} \tau}\right|_{\tau=0} \psi_{j_{k}}^{(k)}(\tau)=\sum_{l=1, l \neq k}^{d} \sum_{j_{1}=1}^{n_{1}} \cdots \sum_{j_{k-1}=1}^{n_{k-1}} \sum_{j_{k+1}=1}^{n_{k+1}} \cdots \sum_{j_{d}=1}^{n_{d}} a_{j_{1}, \ldots, j_{d}} \phi_{j_{1}}^{(1)} \cdots \phi_{j_{l-1}}^{(l-1)} \delta \phi_{j_{l}}^{(l)} \phi_{j_{l+1}}^{(l+1)} \cdots \phi_{j_{\mathrm{d}}}^{(\mathrm{d})}
$$

(and similarly for the terms $\phi_{l_{k}}^{(k)} \psi_{m_{k}}^{(k)}$, etc.), we find that $B^{\prime}(a, \phi) \delta \phi$ is continuous in $\phi$ and

$$
\left\|B^{\prime}(a, \phi) \delta \phi\right\| \leq \kappa\|\delta \phi\|
$$

where $\kappa$ is proportional to $M$ and $\|V\|$. 


\subsection{Lie commutators}

We need bounds for commutators of the nonlinear vector field (considered for fixed coefficients $a$ )

$$
\mathcal{B}(\phi):=B(a, \phi) \phi
$$

with partial differential operators of first and second order. Obviously, the bounds (36) and (37) directly translate into bounds for $\mathcal{B}$,

$$
\begin{aligned}
\|\mathcal{B}(\phi)\| & \leq \widehat{\beta} \\
\left\|\mathcal{B}^{\prime}(\phi) \delta \phi\right\| & \leq \widehat{\kappa}\|\delta \phi\|,
\end{aligned}
$$

with appropriate constants $\widehat{\beta}$ and $\widehat{\kappa}$ depending on $M$, on $\|V\|$, and on a bound of $\|(a, \phi)\|$. Moreover, $\mathcal{B}^{\prime}(\phi) \delta \phi$ depends continuously on $\phi$.

Denote by $\partial_{i}^{(k)}, i=1, \ldots, D_{k}, k=1, \ldots, d$, the partial derivative with respect to the $i$ th spatial coordinate of the $k$ th particle. Consider the Lie commutators

$$
\begin{aligned}
{\left[\partial_{i}^{(k)}, \mathcal{B}\right](\phi) } & =\partial_{i}^{(k)} \mathcal{B}(\phi)-\mathcal{B}^{\prime}(\phi) \partial_{i}^{(k)} \phi \\
{\left[\left(\partial_{i}^{(k)}\right)^{2}, \mathcal{B}\right](\phi) } & =\left(\partial_{i}^{(k)}\right)^{2} \mathcal{B}(\phi)-\mathcal{B}^{\prime}(\phi)\left(\partial_{i}^{(k)}\right)^{2} \phi
\end{aligned}
$$

To indicate the dependence of $\mathcal{B}$ on the potential $V$, we write

$$
\mathcal{B}_{V}=\mathcal{B}
$$

The following key lemma gives nonlinear analogues to the linear commutator relations

$$
\begin{aligned}
{\left[\partial_{i}^{(k)}, V\right] \phi } & =\left(\partial_{i}^{(k)} V\right) \phi \\
{\left[\left(\partial_{i}^{(k)}\right)^{2}, V\right] \phi } & =\left(\left(\partial_{i}^{(k)}\right)^{2} V\right) \phi+2\left(\partial_{i}^{(k)} V\right) \partial_{i}^{(k)} \phi
\end{aligned}
$$

where $\left[\partial_{i}^{(k)}, V\right] \phi=\partial_{i}^{(k)}(V \phi)-V \partial_{i}^{(k)} \phi$ and similarly for $\left(\partial_{i}^{(k)}\right)^{2}$.

Lemma 3.1. If $V$ is twice continuously differentiable with bounded derivatives, then

$$
\begin{aligned}
{\left[\partial_{i}^{(k)}, \mathcal{B}_{V}\right](\phi) } & =\mathcal{B}_{\partial_{i}^{(k)} V}(\phi), \\
{\left[\left(\partial_{i}^{(k)}\right)^{2}, \mathcal{B}_{V}\right](\phi) } & =\mathcal{B}_{\left(\partial_{i}^{(k)}\right)^{2} V}(\phi)+2 \mathcal{B}_{\partial_{i}^{(k)} V}\left(\partial_{i}^{(k)} \phi\right),
\end{aligned}
$$

where $\mathcal{B}_{\partial_{i}^{(k)} V}$ and $\mathcal{B}_{\left(\partial_{i}^{(k)}\right)^{2} V}$ are defined in the same way as $\mathcal{B}_{V}$, but with the potential $V$ replaced by $\partial_{i}^{(k)} V$ and $\left(\partial_{i}^{(k)}\right)^{2} V$, respectively.

Proof. We begin by noting the identities

$$
\begin{aligned}
\mathcal{B}^{\prime}(\phi) \partial_{i}^{(k)} \phi & =\left.\frac{\mathrm{d}}{\mathrm{d} \tau}\right|_{\tau=0} \mathcal{B}\left(\mathrm{e}^{\tau \partial_{i}^{(k)}} \phi\right) \\
\mathrm{i} \mathcal{B}^{\prime}(\phi)\left(\partial_{i}^{(k)}\right)^{2} \phi & =\left.\frac{\mathrm{d}}{\mathrm{d} \tau}\right|_{\tau=0} \mathcal{B}\left(\mathrm{e}^{\mathrm{i} \tau\left(\partial_{i}^{(k)}\right)^{2}} \phi\right),
\end{aligned}
$$


where $\mathrm{e}^{\tau \partial_{i}^{(k)}}$ and $\mathrm{e}^{\mathrm{i} \tau\left(\partial_{i}^{(k)}\right)^{2}}$ denote the unitary groups generated by the skew-adjoint operators $\partial_{i}^{(k)}$ and $\mathrm{i}\left(\partial_{i}^{(k)}\right)^{2}$, respectively. To compute the respective commutators, we use the identity

$$
\begin{aligned}
\left.\frac{\mathrm{d}}{\mathrm{d} \tau}\right|_{\tau=0}\left\langle\mathrm{e}^{\tau \partial_{i}^{(k)}} \phi_{j_{k}}^{(k)}|V| \mathrm{e}^{\tau \partial_{i}^{(k)}} \phi_{l_{k}}^{(k)}\right\rangle & =\left.\frac{\mathrm{d}}{\mathrm{d} \tau}\right|_{\tau=0}\left\langle\phi_{j_{k}}^{(k)} \mid \mathrm{e}^{-\tau \partial_{i}^{(k)}} V \mathrm{e}^{\tau \partial_{i}^{(k)}} \phi_{l_{k}}^{(k)}\right\rangle \\
=-\left\langle\phi_{j_{k}}^{(k)} \mid\left[\partial_{i}^{(k)}, V\right] \phi_{l_{k}}^{(k)}\right\rangle & =-\left\langle\phi_{j_{k}}^{(k)}\left|\partial_{i}^{(k)} V\right| \phi_{l_{k}}^{(k)}\right\rangle .
\end{aligned}
$$

Similarly, it also follows that

$$
\begin{gathered}
\left.\frac{\mathrm{d}}{\mathrm{d} \tau}\right|_{\tau=0}\left\langle\mathrm{e}^{\mathrm{i} \tau\left(\partial_{i}^{(k)}\right)^{2}} \phi_{j_{k}}^{(k)}|V| \mathrm{e}^{\mathrm{i} \tau\left(\partial_{i}^{(k)}\right)^{2}} \phi_{l_{k}}^{(k)}\right\rangle=-\mathrm{i}\left\langle\phi_{j_{k}}^{(k)} \mid\left[\left(\partial_{i}^{(k)}\right)^{2}, V\right] \phi_{l_{k}}^{(k)}\right\rangle \\
=-\mathrm{i}\left\langle\phi_{j_{k}}^{(k)}\left|\left(\partial_{i}^{(k)}\right)^{2} V\right| \phi_{l_{k}}^{(k)}\right\rangle-2 \mathrm{i}\left\langle\phi_{j_{k}}^{(k)}\left|\partial_{i}^{(k)} V\right| \partial_{i}^{(k)} \phi_{l_{k}}^{(k)}\right\rangle .
\end{gathered}
$$

Now, it is straightforward to compute

$$
\begin{aligned}
\left(\partial_{i}^{(k)}\left(B^{(m)}(a, \phi) \phi\right)\right)_{j_{m}}= & 0, \quad j_{m}=1, \ldots, n_{m}, \quad m \neq k \\
\left(\partial_{i}^{(k)}\left(B^{(k)}(a, \phi) \phi\right)\right)_{j_{k}}= & \frac{1}{\mathrm{i}} \sum_{l_{k}=1}^{n_{k}} \sum_{r_{k}=1}^{n_{k}}\left(\rho^{(k)}\right)_{j_{k}, l_{k}}^{-1}\left\langle\psi_{l_{k}}^{(k)}\left|\partial_{i}^{(k)} V\right| \psi_{r_{k}}^{(k)}\right\rangle \phi_{r_{k}}^{(k)} \\
& +\frac{1}{\mathrm{i}} \sum_{j_{k}=1}^{n_{k}} \sum_{l_{k}=1}^{n_{k}} \sum_{r_{k}=1}^{n_{k}}\left(\rho^{(k)}\right)_{j_{k}, l_{k}}^{-1}\left\langle\psi_{l_{k}}^{(k)}|V| \psi_{r_{k}}^{(k)}\right\rangle \partial_{i}^{(k)} \phi_{r_{k}}^{(k)} \\
& -\frac{1}{\mathrm{i}} \sum_{l_{k}=1}^{n_{k}} \sum_{r_{k}=1}^{n_{k}}\left(\rho^{(k)}\right)_{j_{k}, r_{k}}^{-1}\left\langle\phi_{l_{k}}^{(k)} \psi_{r_{k}}^{(k)}|V| u\right\rangle \partial_{i}^{(k)} \phi_{l_{k}}^{(k)}, \quad j_{k}=1, \ldots, n_{k},
\end{aligned}
$$

with $u=\sum_{j_{k}=1}^{n_{k}} \psi_{j_{k}}^{(k)} \phi_{j_{k}}^{(k)}$, and

$$
\begin{aligned}
\left(B^{(m)^{\prime}}(a, \phi) \partial_{i}^{(k)} \phi\right)_{j_{m}}= & -\frac{1}{\mathrm{i}} \sum_{l_{m}=1}^{n_{m}} \sum_{r_{m}=1}^{n_{m}}\left(\rho^{(m)}\right)_{j_{m}, l_{m}}^{-1}\left\langle\psi_{l_{m}}^{(m)}\left|\partial_{i}^{(k)} V\right| \psi_{r_{m}}^{(m)}\right\rangle \phi_{r_{m}}^{(m)} \\
& +\frac{1}{\mathrm{i}} \sum_{l_{m}=1}^{n_{m}} \sum_{r_{m}=1}^{n_{m}}\left(\rho^{(m)}\right)_{j_{m}, r_{m}}^{-1}\left\langle\phi_{l_{m}}^{(m)} \psi_{r_{m}}^{(m)}\left|\partial_{i}^{(k)} V\right| u\right\rangle \phi_{l_{m}}^{(m)}, j_{m}=1, \ldots, n_{m}, m \neq k,(53) \\
\left(B^{(k)^{\prime}}(a, \phi) \partial_{i}^{(k)} \phi\right)_{j_{k}}= & \frac{1}{\mathrm{i}} \sum_{l_{k}=1}^{n_{k}} \sum_{r_{k}=1}^{n_{k}}\left(\rho^{(k)}\right)_{j_{k}, l_{k}}^{-1}\left\langle\psi_{l_{k}}^{(k)}|V| \psi_{r_{k}}^{(k)}\right\rangle \partial_{i}^{(k)} \phi_{r_{k}}^{(k)} \\
& +\frac{1}{\mathrm{i}} \sum_{l_{k}=1}^{n_{k}} \sum_{r_{k}=1}^{n_{k}}\left(\rho^{(k)}\right)_{j_{k}, r_{k}}^{-1}\left\langle\phi_{l_{k}}^{(k)} \psi_{r_{k}}^{(k)}\left|\partial_{i}^{(k)} V\right| u\right\rangle \phi_{l_{k}}^{(k)} \\
& -\frac{1}{\mathrm{i}} \sum_{l_{k}=1}^{n_{k}} \sum_{r_{k}=1}^{n_{k}}\left(\rho^{(k)}\right)_{j_{k}, r_{k}}^{-1}\left\langle\phi_{l_{k}}^{(k)} \psi_{r_{k}}^{(k)}|V| u\right\rangle \partial_{i}^{(k)} \phi_{l_{k}}^{(k)}, \quad j_{k}=1, \ldots, n_{k} .
\end{aligned}
$$

Combining (51) and (53), and (52) and (54), respectively, we obtain (45). An analogous computation using (50) yields (46). 


\subsection{The mild solution}

In order to prove that the system (29)-(30) with initial data $\left(a_{0}, \phi_{0}\right)$ has a unique classical solution, we first prove the existence of a unique mild solution ( $c f .[22]$ ), that is, of a solution of the system of integral equations

$$
\begin{aligned}
& a(t)=a_{0}+\int_{0}^{t} A(\phi(s)) a(s) \mathrm{d} s \\
& \phi(t)=\mathrm{e}^{-\mathrm{i} t T} \phi_{0}+\int_{0}^{t} \mathrm{e}^{-\mathrm{i}(t-s) T} B(a(s), \phi(s)) \phi(s) \mathrm{d} s .
\end{aligned}
$$

Here, (56) results from a formal application of the variation of constant formula with the unitary evolution operator $\mathrm{e}^{-\mathrm{i} t T}$ of the free-particle Schrödinger equation.

The existence of a unique mild solution $t \mapsto(a(t), \phi(t)) \in C\left(\left[0, t_{0}\right], \mathbb{C}^{N} \times\left(L^{2}\right)^{n}\right)$, for sufficiently small $t_{0}>0$, is obtained from a contraction argument for the fixed-point iteration on (55)-(56). Standard arguments, using the bounds and Lipschitz bounds (34) and (39), (40), yield a contraction on a closed ball around $t \mapsto\left(a_{0}, \mathrm{e}^{-\mathrm{i} t T} \phi_{0}\right)$ in $C\left(\left[0, t_{0}\right], \mathbb{C}^{N} \times\left(L^{2}\right)^{n}\right)$, for some $t_{0}>0$ that depends on $\|V\|$ and $\left\|\rho^{-1}\left(a_{0}\right)\right\|$, and on $\left\|\left(a_{0}, \phi_{0}\right)\right\|$. The solution can thus be continued onto any interval $\left[0, t_{0}\right]$ on which $\left\|\rho^{-1}(a(t))\right\|$ and $\|(a(t), \phi(t))\|$ remain bounded.

Since the solution $a$ then satisfies the differential equation (29) with the skew-hermitian matrix $A(\phi)$, it follows that $a$ is of constant norm:

$$
\|a(t)\|=\left\|a_{0}\right\|=1 .
$$

Later we will see that also $\|\phi(t)\|=1$ for all times, but this cannot yet be concluded at the present stage. Once this fact is established, by the above construction we can extend the mild solution to the interval $\left[0, t^{*}\right)$ with $t^{*}$ as given in Theorem 2.1 .

\subsection{Mollifiers}

We recapitulate some properties of the regularisation of functions by convolution with a mollifier. For more details and proofs we refer, e.g., to [1]. For $x \in \mathbb{R}^{D}$ and $\varepsilon>0$, consider the mollifier

$$
\mu_{\varepsilon}(x)=\frac{1}{\varepsilon^{D}} \mu\left(\frac{x}{\varepsilon}\right) \quad \text { with } \quad \mu(x)=\frac{1}{(2 \pi)^{D / 2}} \mathrm{e}^{-|x|^{2} / 2} .
$$

The scaling is such that $\int_{\mathbb{R}^{D}} \mu_{\varepsilon}(x) \mathrm{d} x=\int_{\mathbb{R}^{D}} \mu(x) \mathrm{d} x=1$. The convolution

$$
\left(\mu_{\varepsilon} * f\right)(x)=\int_{\mathbb{R}^{D}} \mu_{\varepsilon}(x-y) f(y) \mathrm{d} y
$$

satisfies the following properties:

(R1) If $f \in L^{2}$, then $\mu_{\varepsilon} * f \in L^{2}$ and $\left\|\mu_{\varepsilon} * f\right\| \leq\|f\|$.

(R2) For $f \in L^{2}, \lim _{\varepsilon \rightarrow 0}\left\|\mu_{\varepsilon} * f-f\right\|=0$.

(R3) If $f \in L^{2}$, then $\mu_{\varepsilon} * f \in H^{m}$ for all $m \geq 1$, and $\partial_{i}^{(k)}\left(\mu_{\varepsilon} * f\right)=\left(\partial_{i}^{(k)} \mu_{\varepsilon}\right) * f \in L^{2}$, and similarly for partial derivatives of higher order.

(R4) If $f \in H^{1}$, then $\partial_{i}^{(k)}\left(\mu_{\varepsilon} * f\right)=\mu_{\varepsilon} *\left(\partial_{i}^{(k)} f\right)$.

(R5) For $f \in L^{2}, \mu_{\varepsilon} *\left(\mathrm{e}^{-\mathrm{i} t T} f\right)=\mathrm{e}^{-\mathrm{i} t T}\left(\mu_{\varepsilon} * f\right)$.

\subsection{Regularisation of the mild solution}

To prove that the mild solution $(a, \phi)$ computed from (55)-(56) is indeed a classical solution of (29)-(30), we need to show $H^{2}$ regularity of $\phi$ as defined by (56). We may take the coefficient vector $a$ as given by the unique solution of $(55)-(56)$, of which we recall $(57)$. 
Before showing the regularity of $\phi$, we define an auxiliary function $\phi_{\varepsilon}$ by means of the mollifier introduced above. We define $\phi_{\varepsilon}$ as the solution of the system of integral equations

$$
\phi_{\varepsilon}(t)=\mu_{\varepsilon} *\left(\mathrm{e}^{-\mathrm{i} t T} \phi_{0}+\int_{0}^{t} \mathrm{e}^{-\mathrm{i}(t-s) T} \mathcal{B}\left(\phi_{\varepsilon}(s)\right) \mathrm{d} s\right)
$$

where we write $\mathcal{B}\left(\phi_{\varepsilon}(s)\right)=B\left(a(s), \phi_{\varepsilon}(s)\right) \phi_{\varepsilon}(s)$ (we suppress the additional dependence on $s$ through $a(s)$ in the notation, since it is irrelevant in the following arguments and clear from the context). The unique existence of $t \mapsto \phi_{\varepsilon}(t)$ in $C\left(\left[0, t_{0}\right],\left(L^{2}\right)^{n}\right)$ is obtained by fixed point iteration similarly to Section 3.4. By (R3) from Section 3.5, we have $\phi_{\varepsilon}$ in $H^{2}$. Note that $\mu_{\varepsilon} * \phi_{0} \rightarrow \phi_{0}$ in $L^{2}$ due to (R2) from Section 3.5. Next we show that

$$
\lim _{\varepsilon \rightarrow 0} \max _{t \in\left[0, t_{0}\right]}\left\|\phi_{\varepsilon}(t)-\phi(t)\right\|=0 .
$$

From the definitions (56) and (59) we obtain

$$
\begin{aligned}
\left\|\phi_{\varepsilon}(t)-\phi(t)\right\| & =\left\|\mu_{\varepsilon} *\left(\mathrm{e}^{-\mathrm{i} t T} \phi_{0}+\int_{0}^{t} \mathrm{e}^{-\mathrm{i}(t-s) T} \mathcal{B}\left(\phi_{\varepsilon}(s)\right) \mathrm{d} s\right)-\left(\mathrm{e}^{-\mathrm{i} t T} \phi_{0}+\int_{0}^{t} \mathrm{e}^{-\mathrm{i}(t-s) T} \mathcal{B}(\phi(s)) \mathrm{d} s\right)\right\| \\
& \leq\left\|\mathrm{e}^{-\mathrm{i} t T}\left(\mu_{\varepsilon} * \phi_{0}-\phi_{0}\right)\right\|+\left\|\int_{0}^{t} \mathrm{e}^{-\mathrm{i}(t-s) T}\left(\mu_{\varepsilon} * \mathcal{B}\left(\phi_{\varepsilon}(s)\right)-\mathcal{B}(\phi(s))\right) \mathrm{d} s\right\| \\
& \leq\left\|\mu_{\varepsilon} * \phi_{0}-\phi_{0}\right\|+\int_{0}^{t}\left\|\mu_{\varepsilon} * \mathcal{B}(\phi(s))-\mathcal{B}(\phi(s))\right\| \mathrm{d} s+\int_{0}^{t}\left\|\mu_{\varepsilon} *\left(\mathcal{B}\left(\phi_{\varepsilon}(s)\right)-\mathcal{B}(\phi(s))\right)\right\| \mathrm{d} s \\
& \leq M_{\varepsilon}+\widehat{\kappa} \int_{0}^{t}\left\|\phi_{\varepsilon}(s)-\phi(s)\right\| \mathrm{d} s,
\end{aligned}
$$

where $M_{\varepsilon} \rightarrow 0$ as $\varepsilon \rightarrow 0$ by (R2) in Section 3.5 and by the dominated convergence theorem. We have used the fact that $\mathrm{e}^{-\mathrm{i} t T}$ is a unitary group of operators, the properties (R5) and (R1) from Section 3.5, and the bound (40). Using the Gronwall inequality, we can now conclude (60).

\section{7. $H^{1}$ regularity}

We now assume that the components of the initial data $\phi_{0}$ are in $H^{1}$, and $V$ is continuously differentiable with bounded derivative. We need to show that $t \mapsto \phi(t)$ is a continuous function from $\left[0, t_{0}\right]$ to $H^{1}$, or equivalently, that $t \mapsto \partial_{i}^{(k)} \phi(t)$ are continuous $L^{2}$-valued functions. Formal differentiation of the integral equation (56) and use of the relation $\partial_{i}^{(k)} \mathrm{e}^{-\mathrm{i} t T} \phi=\mathrm{e}^{-\mathrm{i} t T} \partial_{i}^{(k)} \phi$ for $\phi$ in $H^{1}$ would yield the following relation for $\partial_{i}^{(k)} \phi(t)$ :

$$
\begin{aligned}
\partial_{i}^{(k)} \phi(t) & =\mathrm{e}^{-\mathrm{i} t T} \partial_{i}^{(k)} \phi_{0}+\int_{0}^{t} \mathrm{e}^{-\mathrm{i}(t-s) T} \partial_{i}^{(k)} \mathcal{B}(\phi(s)) \mathrm{d} s \\
& =\mathrm{e}^{-\mathrm{i} t T} \partial_{i}^{(k)} \phi_{0}+\int_{0}^{t} \mathrm{e}^{-\mathrm{i}(t-s) T}\left(\mathcal{B}^{\prime}(\phi(s)) \partial_{i}^{(k)} \phi(s)+\left[\partial_{i}^{(k)}, \mathcal{B}\right](\phi(s))\right) \mathrm{d} s
\end{aligned}
$$

where we have used the definition (41) of the commutator. After substituting the commutator according to Lemma 3.1, we take this formal equation as a motivation to consider the integral equation for $\vartheta$ in $C\left(\left[0, t_{0}\right],\left(L^{2}\right)^{n}\right)$,

$$
\vartheta(t)=\mathrm{e}^{-\mathrm{i} t T} \partial_{i}^{(k)} \phi_{0}+\int_{0}^{t} \mathrm{e}^{-\mathrm{i}(t-s) T}\left(\mathcal{B}^{\prime}(\phi(s)) \vartheta(s)+\mathcal{B}_{\partial_{i}^{(k)} V}(\phi(s))\right) \mathrm{d} s .
$$

Using the bound (40) for $\mathcal{B}^{\prime}$ and noting the linearity in $\vartheta$, it is seen that the integral equation (62) has a unique solution $\vartheta \in C\left(\left[0, t_{0}\right],\left(L^{2}\right)^{n}\right)$ with the same $t_{0}$ as in Section 3.4. 
So far, we have shown that unique solutions $\vartheta$ of (62) and $\phi_{\varepsilon}$ of (59) exist, and $\partial_{i}^{(k)} \phi_{\varepsilon}(t)$ is in $L^{2}$. Moreover, the $L^{2}$ convergence (60) holds. Now, we will use these results to show that

$$
\lim _{\varepsilon \rightarrow 0} \max _{t \in\left[0, t_{0}\right]}\left\|\partial_{i}^{(k)} \phi_{\varepsilon}(t)-\vartheta(t)\right\|=0 .
$$

Since differentiation of (59) yields, by (R4) of Section 3.5 and by Lemma 3.1,

$$
\partial_{i}^{(k)} \phi_{\varepsilon}(t)=\mu_{\varepsilon} *\left(\mathrm{e}^{-\mathrm{i} t T} \partial_{i}^{(k)} \phi_{0}+\int_{0}^{t} \mathrm{e}^{-\mathrm{i}(t-s) T}\left(\mathcal{B}^{\prime}\left(\phi_{\varepsilon}(s)\right) \partial_{i}^{(k)} \phi_{\varepsilon}(s)+\mathcal{B}_{\partial_{i}^{(k)} V}\left(\phi_{\varepsilon}(s)\right)\right) \mathrm{d} s\right),
$$

we find that

$$
\begin{aligned}
\left\|\partial_{i}^{(k)} \phi_{\varepsilon}(t)-\vartheta(t)\right\| \leq & \left\|\mu_{\varepsilon} * \partial_{i}^{(k)} \phi_{0}-\partial_{i}^{(k)} \phi_{0}\right\|+\int_{0}^{t}\left\|\mu_{\varepsilon} * \mathcal{B}^{\prime}\left(\phi_{\varepsilon}(s)\right) \partial_{i}^{(k)} \phi_{\varepsilon}(s)-\mathcal{B}^{\prime}(\phi(s)) \vartheta(s)\right\| \mathrm{d} s \\
& +\int_{0}^{t}\left\|\mu_{\varepsilon} * \mathcal{B}_{\partial_{i}^{(k)} V}\left(\phi_{\varepsilon}(s)\right)-\mathcal{B}_{\partial_{i}^{(k)} V}(\phi(s))\right\| \mathrm{d} s \\
\leq & M_{\varepsilon}^{(1)}+\int_{0}^{t}\left\|\mu_{\varepsilon} * \mathcal{B}^{\prime}(\phi(s)) \vartheta(s)-\mathcal{B}^{\prime}(\phi(s)) \vartheta(s)\right\| \mathrm{d} s \\
& +\int_{0}^{t}\left\|\mu_{\varepsilon} *\left(\mathcal{B}^{\prime}\left(\phi_{\varepsilon}(s)\right) \partial_{i}^{(k)} \phi_{\varepsilon}(s)-\mathcal{B}^{\prime}(\phi(s)) \vartheta(s)\right)\right\| \mathrm{d} s \\
& +\int_{0}^{t}\left\|\mu_{\varepsilon} * \mathcal{B}_{\partial_{i}^{(k)} V}(\phi(s))-\mathcal{B}_{\partial_{i}^{(k)} V}(\phi(s))\right\| \mathrm{d} s \\
& +\int_{0}^{t}\left\|\mu_{\varepsilon} *\left(\mathcal{B}_{\partial_{i}^{(k)} V}\left(\phi_{\varepsilon}(s)\right)-\mathcal{B}_{\partial_{i}^{(k)} V}(\phi(s))\right)\right\| \mathrm{d} s \\
\leq & M_{\varepsilon}^{(2)}+C_{1} \int_{0}^{t}\left\|\phi_{\varepsilon}(s)-\phi(s)\right\| \mathrm{d} s+C_{2} \int_{0}^{t}\left\|\partial_{i}^{(k)} \phi_{\varepsilon}(s)-\vartheta(s)\right\| \mathrm{d} s,
\end{aligned}
$$

where $M_{\varepsilon}^{(1)} \rightarrow 0$ and $M_{\varepsilon}^{(2)} \rightarrow 0$ as $\varepsilon \rightarrow 0$. Here we have used the bounds (39) and (40), the continuity of $\mathcal{B}^{\prime}$, and the mollifier properties of Section 3.5. Thus, with (60) and the Gronwall inequality we obtain (63).

We now conclude that $\vartheta=\partial_{i}^{(k)} \phi$ : since $\partial_{i}^{(k)}$ is a closed operator on $L^{2}$, we have

$$
\vartheta(t)=\lim _{\varepsilon \rightarrow 0} \partial_{i}^{(k)} \phi_{\varepsilon}(t)=\partial_{i}^{(k)} \lim _{\varepsilon \rightarrow 0} \phi_{\varepsilon}(t)=\partial_{i}^{(k)} \phi(t)
$$

for the limits with respect to the $L^{2}$ norm. We thus have $\partial_{i}^{(k)} \phi(t) \in\left(L^{2}\right)^{n}$ for all $k, i$, and the proof of the $H^{1}$ regularity of $\phi(t)$ is complete.

\section{8. $H^{2}$ regularity and orthonormality}

The $H^{2}$ regularity follows analogously if we assume that $\phi_{0} \in H^{2}$ and $V$ is twice differentiable with bounded derivatives. In view of a formal differentiation of the integral equation (56) and the second commutator identity in Lemma 3.1, we consider the integral equation

$$
\theta(t)=\mathrm{e}^{-\mathrm{i} t T}\left(\partial_{i}^{(k)}\right)^{2} \phi_{0}+\int_{0}^{t} \mathrm{e}^{-\mathrm{i}(t-s) T}\left(\mathcal{B}^{\prime}(\phi(s)) \theta(s)+\mathcal{B}_{\left(\partial_{i}^{(k)}\right)^{2} V}(\phi(s))+2 \mathcal{B}_{\partial_{i}^{(k)} V}\left(\partial_{i}^{(k)} \phi(s)\right)\right) \mathrm{d} s .
$$

This integral equation has a unique, continuous solution $\theta \in C\left(\left[0, t_{0}\right],\left(L^{2}\right)^{n}\right)$ (again with the same $t_{0}$ where the mild solution exists), which depends $L^{2}$ Lipschitz continuously on the initial data $\left(\partial_{i}^{(k)}\right)^{2} \phi_{0}$. By means of 
the $H^{2}$-regular auxiliary function $\phi_{\varepsilon}$, the solution $\theta$ can be shown to be indeed equal to $\left(\partial_{i}^{(k)}\right)^{2} \phi$. To this end, similar estimates as above can be used, where instead of the commutator identity (45) we use (46) and take into account that $\left(\partial_{i}^{(k)}\right)^{2}$ is likewise a closed operator on $L^{2}$. Altogether we can conclude that $\phi \in C\left(\left[0, t_{0}\right],\left(H^{2}\right)^{n}\right)$. This also implies that $\phi$ is a classical solution of (29)-(30). To see this, we differentiate (55)-(56) on noting that the expression $T \phi$ is well-defined since $\phi$ is in $H^{2}$. This yields that $t \mapsto(a(t), \phi(t))$ is continuously differentiable with time derivatives in $L^{2}$ and satisfies the system of differential equations (29)-(30).

Now, since $(a, \phi)$ defined by (55)-(56) satisfies (29)-(30), we can show that $\phi$ also satisfies $(25)$ with $G^{(k)}=$ $T^{(k)}$ : First, note that each equation from (30) can be written in the form

$$
\dot{\phi}_{j_{k}}^{(k)}=\frac{1}{\mathrm{i}} T^{(k)} \phi_{j_{k}}^{(k)}+\left(1-P^{(k)}\right) d_{j_{k}}^{(k)}
$$

with a function $d_{j_{k}}^{(k)} \in L_{x^{(k)}}^{2}$. Consequently,

$$
\begin{aligned}
\left\langle\phi_{j_{k}}^{(k)} \mid \dot{\phi}_{l_{k}}^{(k)}\right\rangle & =\left\langle\phi_{j_{k}}^{(k)} \mid-\mathrm{i} T^{(k)} \phi_{l_{k}}^{(k)}\right\rangle+\left\langle\phi_{j_{k}}^{(k)} \mid\left(1-P^{(k)}\right) d_{l_{k}}^{(k)}\right\rangle=\left\langle\phi_{j_{k}}^{(k)} \mid-\mathrm{i} T^{(k)} \phi_{l_{k}}^{(k)}\right\rangle+\left\langle\left(1-P^{(k)}\right) \phi_{j_{k}}^{(k)} \mid d_{l_{k}}^{(k)}\right\rangle \\
& =-\mathrm{i}\left\langle\phi_{j_{k}}^{(k)}\left|T^{(k)}\right| \phi_{l_{k}}^{(k)}\right\rangle,
\end{aligned}
$$

since $\left(1-P^{(k)}\right)$ is self-adjoint. By $(24)$, this further implies that $\phi_{j_{k}}^{(k)}$ satisfy the orthonormality relation (12), provided that the initial data are orthonormal. As we have noted previously in Section 3.4, this now implies that the solution can actually be extended up to $t^{*}$ as given in Theorem 2.1.

Moreover, the formal definition (18) of $\rho^{(k)}$ is now equivalent to (17), and so we conclude that $u=\sum_{J} a_{J} \Phi_{J}$ indeed solves the variational problem (5).

Altogether, this proves the assertions of Theorem 2.1.

\subsection{Higher regularity}

The above arguments can be extended without further difficulty to prove higher regularity of the MCTDH approximation if $V$ is smooth with bounded derivatives and $\phi_{0}$ is regular enough. In this case, commutators for higher spatial differential operators can be computed analogously as in Lemma 3.1. Using bounds for these commutators, it is again possible to show that solutions to the formal integral equations for higher derivatives of $\phi$ exist on $\left[0, t^{*}\right)$, and the respective derivatives of the auxiliary function $\phi_{\varepsilon}$ converge to these, uniformly on compact subintervals. This procedure can be used to show arbitrary regularity of the solution for smooth enough data on the interval $\left[0, t^{*}\right)$, as stated in Corollary 2.2 .

\subsection{Remark: an alternative approach}

The solution of the MCTDH equations is equivalent to the solution of the differential equation on the approximation manifold,

$$
\dot{u}=P(u) \frac{1}{\mathrm{i}} H u=\frac{1}{\mathrm{i}} T u+P(u) \frac{1}{\mathrm{i}} V u, \quad u(0)=u_{0} \in \mathcal{M},
$$

where $P(u)$ denotes the orthogonal projection to $\mathcal{T}_{u} \mathcal{M}$. An elementary calculation shows that

$$
P(u) \frac{1}{\mathrm{i}} V u=\sum_{J} \sum_{K} A_{J, K} a_{K} \Phi_{J}+\sum_{k=1}^{d} \sum_{j_{k}=1}^{n_{k}} \sum_{l_{k}=1}^{n_{k}} B_{j_{k}, l_{k}}^{(k)} \phi_{l_{k}}^{(k)} \psi_{j_{k}}^{(k)} .
$$


However, this problem formulation does not lend itself to an analysis as conveniently as in the above approach, even though similar terms have to be estimated. Combining the coordinate-free evolution equation with the necessary estimates of the coordinate functions $(a, \phi)$ in the analysis appears to lead to more technical complications than working with the differential equations for $(a, \phi)$ right away and returning to (65) at the very end.

\section{Conclusions and outlook}

In this paper, we have analysed the regularity of the approximation to the solution of the time-dependent Schrödinger equation defined by the multi-configuration time-dependent Hartree method when the potential defining the Hamiltonian is sufficiently smooth. Using bounds for the Lie commutators of the vector field with differential operators, it was shown that a unique solution $(a, \phi)$ to the MCTDH equations exists in the Sobolev space $H^{m}$ if the initial data is in $H^{m}(m \geq 2)$, and the solution depends on the initial data Lipschitz continuously with respect to the $H^{m}$ norm. This regularity extends directly to the associated approximation to the wave function defined by the Dirac-Frenkel variational equations. The well-posedness is ensured for all times where the density matrices $\rho^{(k)}$ remain nonsingular.

The $H^{2}$ regularity has important consequences for the approximation properties of the MCTDH approach. According to [18], Theorem 4.1, the error of the MCTDH approximation to the exact wave function is - for sufficiently short time intervals - of the same order of magnitude as the error of the best approximation in the approximation manifold. This result requires $H^{2}$ regularity of the approximate wave function.

The regularity is also needed to ensure good performance of numerical methods used to solve the MCTDH equations, both for space discretisation and for the variational splitting integrator of [17] for the discretisation in time. The commutator identities shown in the course of our proofs are likewise an essential ingredient in the error analysis of this time integrator.

An interesting theoretical (though not practically relevant) question concerns the possible convergence of the MCTDH approximations as more and more terms are included in the linear combination of Hartree products. While we cannot exclude that such convergence occurs, we are sceptic that this would hold in general. One might try to base a convergence proof on the above-cited quasi-optimality result of [18], since the best approximation by linear combinations of Hartree products does converge to the exact wave function. However, the approximation bounds of [18] depend on various parameters that explode as the density matrices in the MCTDH method become nearly singular, and this is just what must be expected to happen as more and more nearly irrelevant terms are included in the MCTDH approximation. Given the possible lack of convergence of MCTDH, it is nonetheless safe to consider MCTDH as a highly successful model reduction technique (analogous to low-rank model reductions in other fields) which for a fixed number of Hartree products leads to equations of motion with a sound existence and regularity theory as developed in this paper, with a quasi-optimality property linking the MCTDH approximation to the best approximation of the wave function by the given number of Hartree products (at least on short time intervals), and complemented with efficient numerical techniques for the solution of the MCTDH equations of motion.

It is worthwhile to note that the regularity results proven in this paper remain valid for variants and extensions of the MCTDH method designed for the computational treatment of larger systems, such as the coupling with Gaussian wavepackets for secondary modes [7] and the multi-mode, cascadic or multilayer versions of MCTDH $[4,25]$ with which spin-boson systems with up to 500 degrees of freedom have been treated.

Our results apply also to the multi-configuration time-dependent Hartree-Fock (MCTDHF) method for the electronic Schrödinger equation, if the potential is smooth. In [15] it was demonstrated that the antisymmetry assumption inherent in MCTDHF implies that only a subset of the full MCTDH equations (29)-(30) have to be solved, but the problem formulation is not altered in another way. Thus, we can conclude that there exists a unique, regular solution of MCTDHF, if a nonsingular, screened Coulomb potential is used. This constitutes a commonly used regularisation of the singular Coulomb potential. It would be interesting to clarify the regularity properties in the case of the singular Coulomb potential as well. 


\section{REFERENCES}

[1] H.W. Alt, Lineare Funktionalanalysis. Springer Verlag, Berlin-Heidelberg-New York, 3rd edition (1999).

[2] M. Baer and G.D. Billing Eds., The Role of Degenerate States in Chemistry, Advances in Chemical Physics 124, Wiley (2002).

[3] M.H. Beck and H.-D. Meyer, An efficient and robust integration scheme for the equations of the multiconfiguration timedependent Hartree (MCTDH) method. Z. Phys. D 42 (1997) 113-129.

[4] M.H. Beck, A. Jäckle, G.A. Worth and H.-D. Meyer, The multiconfiguration time-dependent Hartree (MCTDH) method: a highly efficient algorithm for propagating wavepackets. Phys. Rep. 324 (2000) 1-105.

[5] A. Bove, G. Da Prato and G. Fano, An existence proof for the Hartree-Fock time-dependent problem with bounded two-body interaction. Comm. Math. Phys. 37 (1974) 183-191.

[6] A. Bove, G. Da Prato and G. Fano, On the Hartree-Fock time-dependent problem. Comm. Math. Phys. 49 (1976) 25-33.

[7] I. Burghardt, H.-D. Meyer and L.S. Cederbaum, Approaches to the approximate treatment of complex molecular systems by the multiconfiguration time-dependent Hartree method. J. Chem. Phys. 111 (1999) 2927-2939.

[8] J. Caillat, J. Zanghellini, M. Kitzler, W. Kreuzer, O. Koch and A. Scrinzi, Correlated multielectron systems in strong laser pulses - an MCTDHF approach. Phys. Rev. A 71 (2005) 012712.

[9] J.M. Chadam and R.T. Glassey, Global existence of solutions to the Cauchy problem for time-dependent Hartree equations. J. Math. Phys. 16 (1975) 1122-1130.

[10] P.A.M. Dirac, Note on exchange phenomena in the Thomas atom. Proc. Cambridge Phil. Soc. 26 (1930) 376-385.

[11] W. Domcke, D.R. Yarkony and H. Köppel Eds., Conical Intersections. Electronic Structure, Dynamics 83 Spectroscopy. World Scientific, Singapore, 2004.

[12] E. Faou and C. Lubich, A Poisson integrator for Gaussian wavepacket dynamics. Comput. Visual. Sci. 9 (2005) $45-55$.

[13] J. Frenkel, Wave Mechanics, Advanced General Theory. Clarendon Press, Oxford (1934).

[14] G. Friesecke, The multiconfiguration equations for atoms and molecules: charge quantization and existence of solutions. Arch. Ration. Mech. Anal. 169 (2003) 35-71.

[15] O. Koch, W. Kreuzer and A. Scrinzi, Approximation of the time-dependent electronic Schrödinger equation by MCTDHF. Appl. Math. Comput. 173 (2006) 960-976.

[16] M. Lewin, Solutions of the multiconfiguration equations in quantum chemistry. Arch. Ration. Mech. Anal. 171 (2004) 83-114.

[17] C. Lubich, A variational splitting integrator for quantum molecular dynamics. Appl. Numer. Math. 48 (2004) 355-368.

[18] C. Lubich, On variational approximations in quantum molecular dynamics. Math. Comp. 74 (2005) 765-779.

[19] A.D. McLachlan, A variational solution of the time-dependent Schrödinger equation. Mol. Phys. 8 (1964) 39-44.

[20] H.-D. Meyer and G.A. Worth, Quantum molecular dynamics: propagating wavepackets and density operators using the multiconfiguration time-dependent Hartree (MCTDH) method. Theo. Chem. Acc. 109 (2003) 251-267.

[21] H.-D. Meyer, U. Manthe and L.S. Cederbaum, The multi-configurational time-dependent Hartree approach. Chem. Phys. Lett. 165 (1990) 73-78.

[22] A. Pazy, Semigroups of Linear Operators and Applications to Partial Differential Equations. Springer-Verlag, New York (1983).

[23] A. Raab, G.A. Worth, H.-D. Meyer and L.S. Cederbaum, Molecular dynamics of pyrazine after excitation to the $S_{2}$ electronic state using a realistic 24-mode model Hamiltonian. J. Chem. Phys. 110 (1999) 936-946.

[24] D.J. Rowe, A. Ryman and G. Rosensteel, Many-body quantum mechanics as a symplectic dynamical system. Phys. Rev. A 22 (1980) 2362-2373.

[25] H. Wang and M. Thoss, Multilayer formulation of the multiconfiguration time-dependent Hartree theory. J. Chem. Phys. 119 (2003) 1289-1299. 